\title{
Teaching the Principles of Massive MIMO: Exploring reciprocity-based multiuser MIMO beamforming using acoustic waves
}

\author{
Erik G Larsson, Danyo Danev, Mikael Olofsson and Simon Sörman \\ Journal Article
}

Tweet

N.B.: When citing this work, cite the original article.

(C)2016 IEEE. Personal use of this material is permitted. However, permission to reprint/republish this material for advertising or promotional purposes or for creating new collective works for resale or redistribution to servers or lists, or to reuse any copyrighted component of this work in other works must be obtained from the IEEE.

Erik G Larsson, Danyo Danev, Mikael Olofsson and Simon Sörman, Teaching the Principles of Massive MIMO, IEEE signal processing magazine (Print), 2017. 34(1), pp.40-47.

http://dx.doi.org/10.1109/MSP.2016.2618914

Postprint available at: Linköping University Electronic Press

http://urn.kb.se/resolve?urn=urn:nbn:se:liu:diva-136361

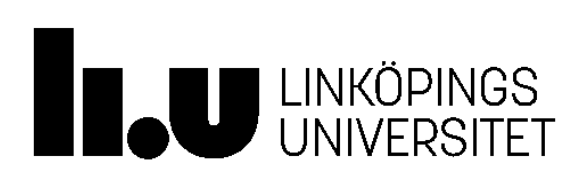




\section{Teaching the Principles of Massive MIMO}

October 17, 2016

Authors: Erik G. Larsson ${ }^{1}$, Danyo Danev ${ }^{1}$, Mikael Olofsson ${ }^{1}$ and Simon Sörman ${ }^{2}$

${ }^{1}$ Linköping University, Dept. of Electrical Engineering (ISY), 58183 Linköping, Sweden

${ }^{2}$ Ericsson Research, 58183 Linköping, Sweden (former student in the project course)

\section{Introduction}

Massive MIMO is currently the most compelling wireless physical layer technology, and a key component of $5 \mathrm{G}$ systems. The understanding of its core principles has emerged during the last five years, and material is becoming available that is rigorously refined to focus on timeless fundamentals [1], facilitating the instruction of the topic to both master- and doctoral-level students [2]. Meaningful laboratory work that exposes the operational principles of Massive MIMO is more difficult to accomplish. At LiU, this was achieved through a project course, based on the conceive-design-implement-operate (CDIO) concept [3], and through the creation of a specially designed experimental setup using acoustic signals.

The course was developed with the following three objectives in mind:

- Exposure of students to emerging concepts and to the technology of the future, not of the past. This target was inherently met via the focus on Massive MIMO.

- Promotion of a systems view of thinking, requiring the synthesis of knowledge acquired through the classical curriculum. This goal was achieved through the development of a unique acoustic, reciprocity-based Massive MIMO laboratory setup (Figure 1), with students taking lead both in its development, evaluation and subsequent refinement.

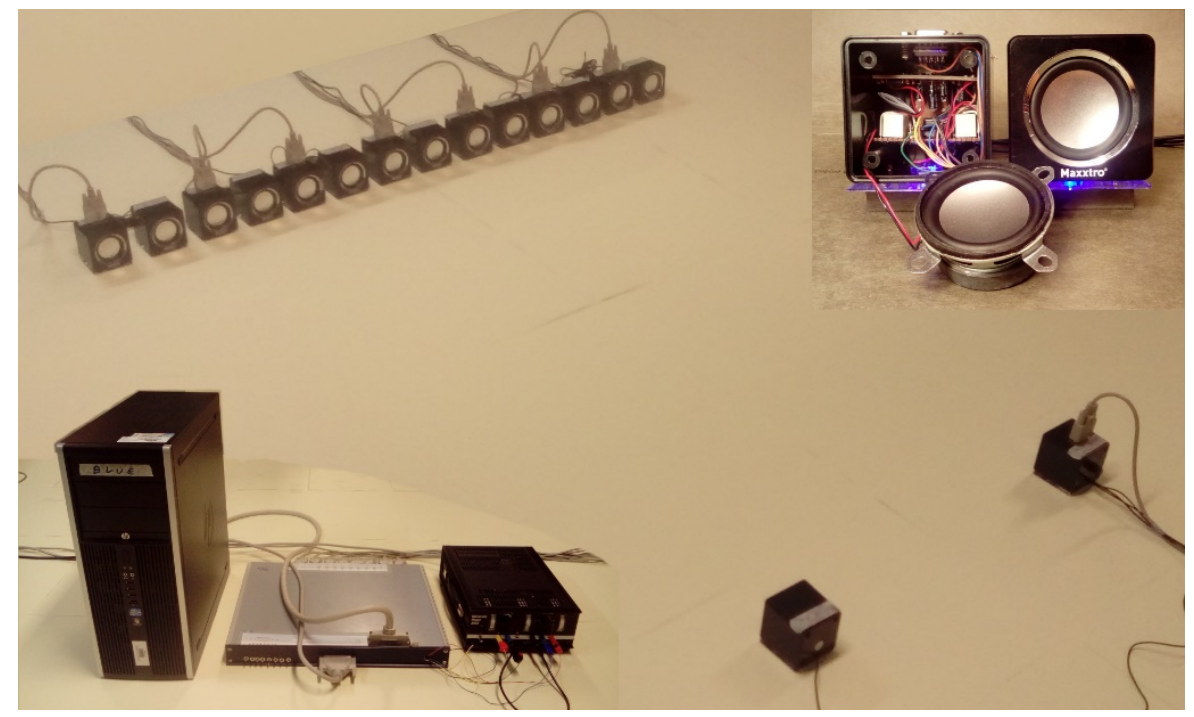

Figure 1. Massive MIMO laboratory setup at LiU 
- Fosterage of genuine teamwork, preparing students for a dynamic work environment. This was efficiently facilitated through the adoption of the CDIO project concept, which integrates technical work with the instruction of project management and entrepreneurial skills.

\section{Massive MIMO: The Scalable 5G Wireless Access Technology}

Massive MIMO exploits the use of large antenna arrays at wireless base stations to simultaneously serve a large number of autonomous terminals through spatial multiplexing. The multiplexing takes the form of beamforming, also known as multiuser precoding, effectively creating transmitted signals that add up constructively on the spots where the terminals are located and destructively almost everywhere else (Figure 2).

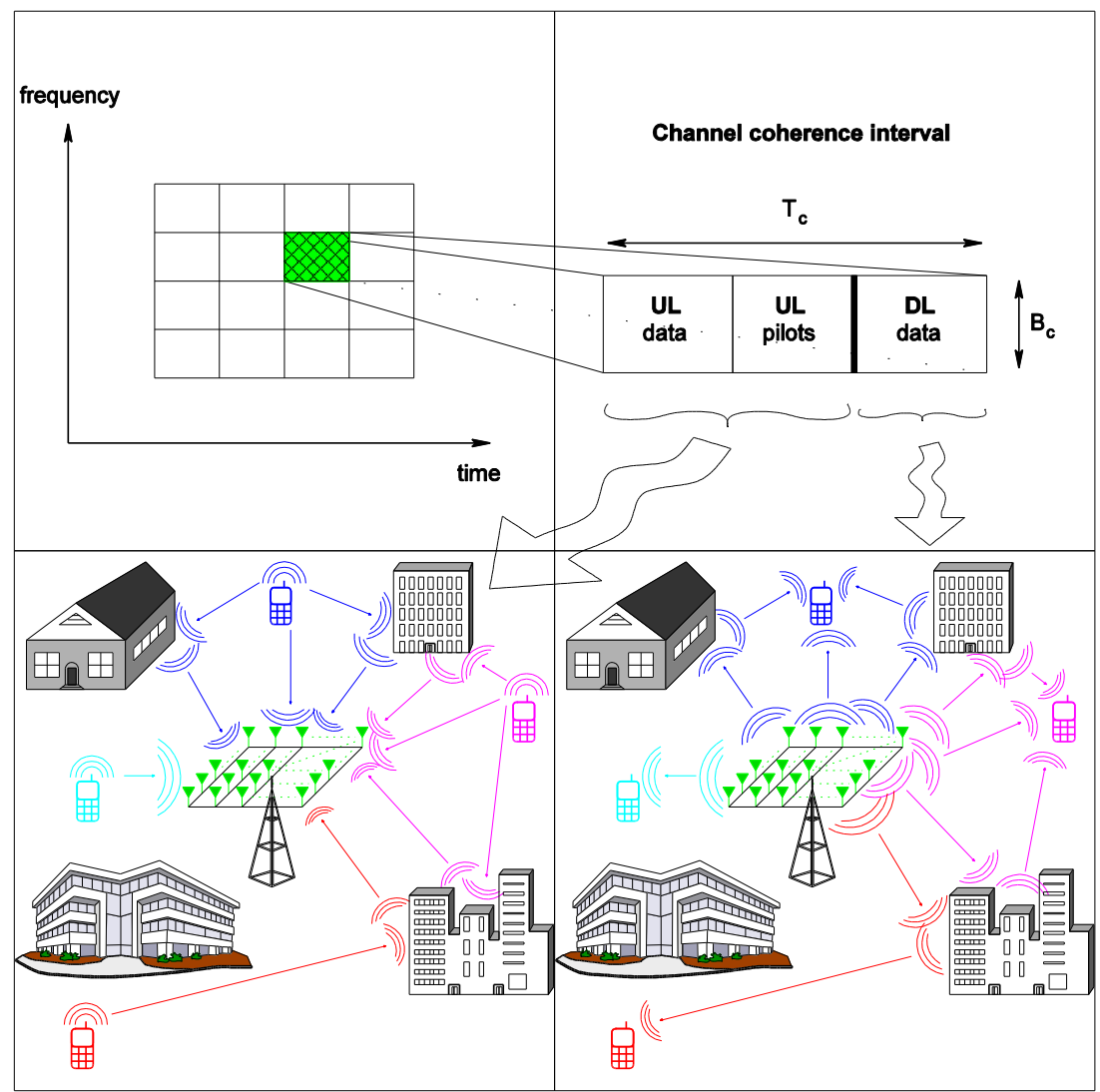

Figure 2. Massive MIMO

Being the ultimately most useful and scalable form of Multiuser MIMO, Massive MIMO departs from conventional MIMO technology in several ways:

- The acquisition of accurate instantaneous channel state information (CSI) at the base station is facilitated through time-division duplexing operation, and the transmission of pilot waveforms by the terminals. On uplink, terminals transmit pilots and payload; subsequently on downlink, the base station beamforms to the terminals. Reciprocity of uplink-downlink propagation is essential to the use of uplink CSI for downlink precoding, and achieved in practice through calibration of the RF chains [4]. The reliance on reciprocity permits accurate channel training even in highway-speed mobility scenarios. 
- Extraordinary spectral efficiencies are achieved [5], although no attempt is made to operate at the Shannon limit. The base station uses linear signal processing, and the terminal uses almost no signal processing at all. CSI is acquired only at the base station, thence obviating the need for downlink pilots. This renders Massive MIMO entirely scalable with respect to the number of base station antennas.

- Each terminal is assigned the full bandwidth. Thanks to channel hardening, the effective channel gain for each terminal is deterministic and frequency- (subcarrier-) independent, which greatly simplifies resource allocation problems, and facilitates simple closed-form solutions for power control.

- Different base stations do not cooperate, other than for "slow" tasks such as power control and pilot sequence assignment. Macro-diversity against shadow fading is accomplished by appropriate terminal-to-base station association; the large number of spatial degrees of freedom guarantees that every base station has room to accommodate additional terminals with very high probability.

- By virtue of the large array gain and the ability to null out interference, max-min fairness power control is feasible, and can be exploited to yield uniform quality of service within each cell.

\section{Communications Laboratory Exercises: RF versus Acoustic}

Laboratory work that uses communications over $\mathrm{RF}$ in general requires substantial equipment investments, the availability of a sufficiently interference-free environment (alternatively spectrum licenses for experimental operation), the use of high sampling rates which in turn generates large quantities of baseband data, and the access to highperformance measurement equipment for calibration and debugging purposes. Owing to precise requirements on the phase reference distribution, these difficulties are significantly accentuated when going from single-antenna to multiple-antenna setups. Consequently, experimental activities on Massive MIMO are concentrated to research-grade testbeds that require the investment of many person-years to build, and permanent engineering staff to maintain --- thus inherently unsuitable for instructional purposes. Worldwide, only few such testbeds, operational and under construction, are known $[5,6,7,8,9,10]$.

The basic physics of wave propagation is substantially the same for electromagnetic wave propagation and for acoustic wave propagation, disregarding polarization aspects. Consequently, wireless communications course laboratory work may use sound waves instead of RF, which for point-to-point communications requires only a loudspeaker as transmitter and a microphone as receiver. The wavelength of sound in the audible regime is comparable to the wavelength of radio in the $\mathrm{GHz}$ regime; hence the channel coherence time for acoustic communication indoors is sufficiently long to easily permit real-time experimentation over a time-invariant channel. Additionally, many phenomena such as small-scale fading can be observed with an acoustic setup as well. The low bandwidth of audible sound results in low sampling rates, modest requirements on clock synchronization, and manageable amounts of baseband data. These features are routinely exploited in many university course laboratory exercises. 


\section{Teaching MIMO, and Massive MIMO Principles in the Lab}

Laboratory work on point-to-point wireless communications is straightforward. In contrast, serious experimentation with Massive MIMO concepts is a less obvious task, owing to the need for many simultaneously operating transceivers and the uplink-downlink reciprocity requirement. The experimental setup developed in our course addressed this difficulty as follows:

- A loudspeaker element natively functions as sound transmitter when fed with an amplified input signal. It was experimentally revealed that oppositely, it also can function well as a receiver (microphone), by connecting it to a high-input-impedance amplifier and measuring the resulting signal.

- A set of off-the-shelf loudspeaker elements was acquired and redesigned as follows:

1. the built-in amplifier chain was retained, yielding the transmit branch.

2. an amplifier was installed in order to use the loudspeaker element as microphone, yielding the receive branch.

3. a relay that galvanically isolates the transmit and receive branches was installed.

The required additional electronics was fitted inside of the loudspeaker element casing (Figure 1). The rebuilt loudspeaker element, now functioning reciprocally in both the transmit and receive directions, is referred to as "transceiver device" in the following.

- 14 transceiver devices were used to form a base station array and 2 were used to form two independent terminals. All devices were connected to a PC equipped with multi-channel digital-to-analog (D/A) and analog-to-digital (A/D) cards. At a $6.25 \mathrm{kHz}$ sampling rate per channel, the aggregate baseband data rate from all channels combined equaled $1200 \mathrm{~kb} / \mathrm{s}$, facilitating real-time processing in a Matlab environment.

The appendix gives further technical details, and a live demonstration may be found at [11].

\section{Promoting a Systems View of Thinking}

University education traditionally is rather modularized, consisting of courses with a welldefined but often very specific scope. In contrast, practicing engineers must be able to solve problems by synthesis of knowledge received both in formal training and courses, and acquired from experience. The traditional curriculum in communications is no exception: while much time in class is spent on proving capacity theorems and computing error probabilities, most laboratory time (and most engineering efforts) tends to be spent on "making it work": solving practical problems, and integrating components together into systems. Formal training and knowledge of textbook material is indispensable, but real problem solving requires both trial-and-error, and the sourcing of information from books, colleagues, and the Internet.

The experimental setup and its development trained students in every aspect of communications, ranging from the understanding of wave propagation, fundamental Massive MIMO theory, hands-on design and soldering of electronics, software programming and algorithm design. This substantially took the students' understanding of a "system" 
from the input-output box typically taught in signals-and-systems courses, to a "system" meaning a large set of connected, diverse components. Answers were not given to the students but had to be sought through consultation with faculty and senior graduate students. In fact, as the project was untested before, in many cases instructors did not know. A minor risk was taken that the course would not work out, in which case a backup plan was to only perform certain measurements, so that reporting of a minimum level of requirements for the course could be adequately completed.

\section{Providing True Teamwork Experience Through a CDIO Project}

The conceive-design-implement-operate (CDIO) concept [3] was developed by the Massachusetts Institute of Technology in the USA and Chalmers Institute of Technology, Linköping University, and Royal Institute of Technology, all in Sweden, in the late 1990's and the early 2000's. The initial efforts were supported by the Knut and Alice Wallenberg Foundation. Since then more than 120 schools and universities worldwide have joined the CDIO initiative. One of the reasons for the proliferation and success of the concept is that it profoundly facilitates teamwork training for students.

The CDIO framework focuses on teaching of engineering fundamentals from the perspective of real-world systems and products. Through constant input from students, faculty and engineers, the concept has evolved over the years. Currently it represents the state-of-theart in project course organization in engineering schools, preparing the students for the challenges of the professional life in industry.

As one of the founders of the CDIO concept, Linköping University has adopted and implemented the idea from its inception. All engineering Master's programs include a mandatory CDIO course in the curriculum. In collaboration with several global companies that have local offices in Linköping, notably Ericsson and SAAB, a specific project model, LIPS (Linköping Interactive Project Steering) [12], has been developed to support the project management process. The LIPS model introduces a set of documents that the students are required to write during the project, as well as a set of milestones and tollgates that underpin the planning of the project work. In the beginning of the course, students usually express doubts about the necessity of the documentation and the extent of the planning required, but most of them usually appreciate the LIPS project steering model once the course is finished.

The project team normally consists of 4 to 7 students. Generally, these students have not worked together before and an immediate task at the start of the project is to learn to know each other and decide on the different roles that they will have in the project group. In addition to the crucial role of project manager, the group members usually assign a person responsible for documentation, quality, testing, design and customer contact, respectively. The project task is determined in detail by the customer through the definition of a set of requirements that the final product should fulfil. These tasks are usually re-negotiated with the project group until a final set of requirements is agreed upon, a few weeks after the start of the project. The role of the customer is taken by a faculty member and sometimes the course director can take this role. The university also provides support to the students in the form of a supervisor (normally a senior Ph.D. student or teaching assistant) as well as topic 
expertise (from other junior faculty members and Ph.D. students). Figure 3 depicts the various roles and the interactions, with solid lines indicating formal contacts (documents, official meetings) and dashed lines representing informal contacts (advice, meetings). As the project group members work together continuously, the everyday informal contacts between the group members are not shown in the figure.

\section{Project group of students}

Faculty members

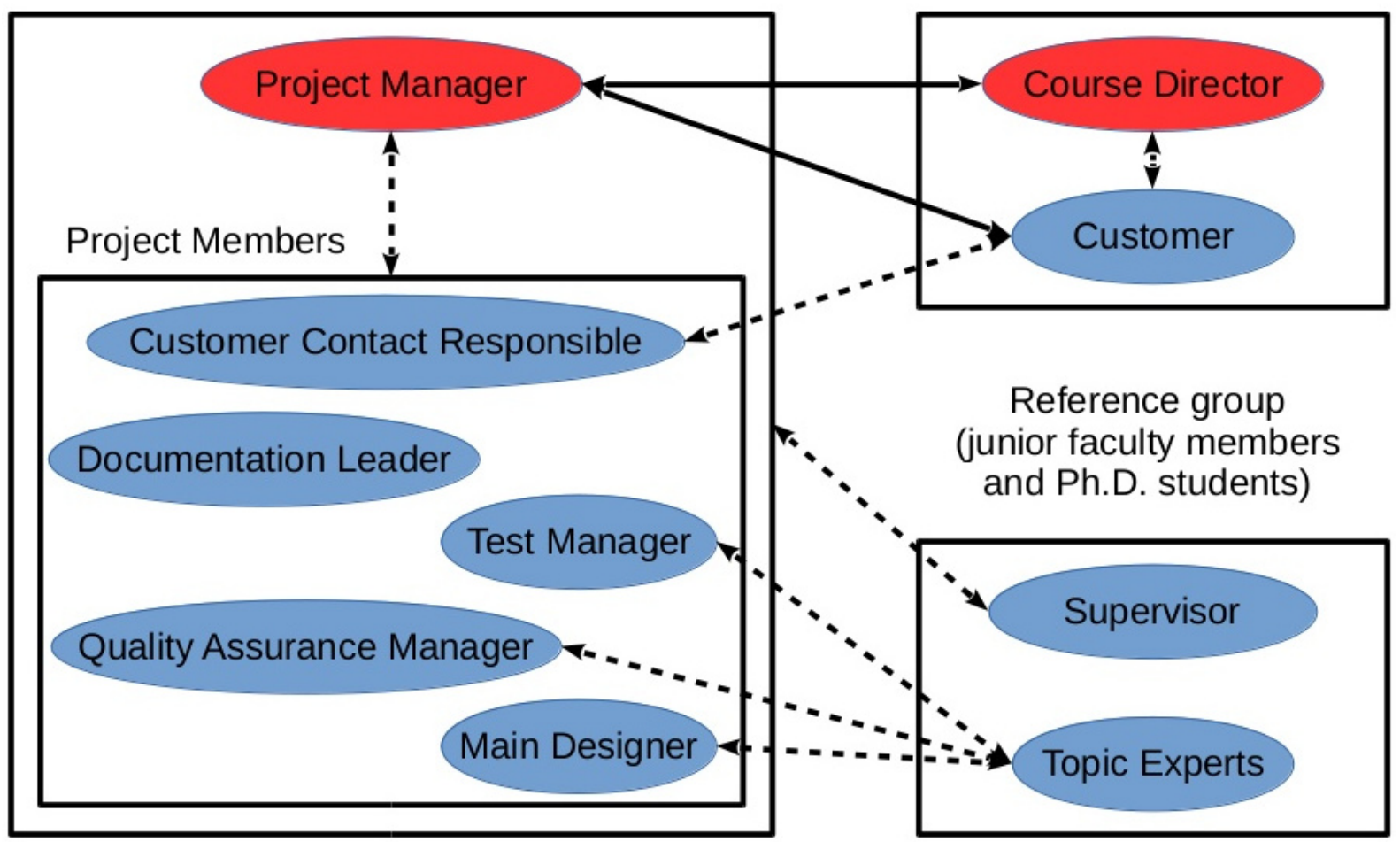

$\longleftrightarrow$ formal contact (official meetings, documentation)

- - informal contacts (meetings, advice)

Figure 3. Roles of the students and faculty members in our implementation of the CDIO project course concept.

Our general experience is that all students involved in these projects have been enthusiastic and have contributed accordingly to their tasks. The group dynamics has varied during the years and has been somewhat dependent on the interests and ambition levels of the participants. Many original ideas from students have been implemented. Most often the desired results have been achieved. Since the time scope for the course is strictly limited to one semester, there is no room for delays. This sometimes has resulted in products that do not reach full functionality. However, in those cases the well documented work has helped subsequent project groups to continue the development and further extend the capabilities of the product.

The purpose of our course in general is to provide a genuine teamwork learning experience in the field of telecommunications. Building an audio test-bed for demonstration of the Massive MIMO concept specifically was a vehicle for bringing the research frontiers to the students and has ignited their interest for the field, as well as prepared them for an industrial career. The combination of hardware and software development is a unique aspect of our course. The challenges of handling hardware imperfections have stimulated 
creative skills of the involved students. Combining all the components and implementing the desired algorithms have required good teamwork, and devotion to the task. The product created so far has shown useful capabilities and is also scalable.

The course project was conducted according to a project model, which rather precisely defines rules and support for the students' work process. This helps the students organize their work in an efficient manner, and make a carefully considered system design before implementing it. The project thus gives the students the opportunity to work in a fashion that is similar to how projects are conducted in industry.

In this course we let the students work very independently, without any initial pointers on how to solve the problem at hand but with encouragement to invent and research potential solutions based on knowledge from previous courses, and with access to expert supervisors who can answer questions and discuss ideas. Our general experience is that students are very successful in forming a working project group, and they enjoy the opportunity to design a system mostly on their own, learning by acquiring hands-on experience.

\section{Lessons Learned and Future Directions}

The experimental acoustic Massive MIMO setup that we have developed is inexpensive and scalable, and the learning-curve for the equipment has been appropriate for the course. The project has been well-received by students in that they can apply their knowledge of communications theory to build a complete working system from scratch. They appreciate the structure of the project in that they get to try out their own ideas in solving a complex problem defined on a relatively high level. They also appreciate the access to expertise when help is needed. The students have expressed that they enjoy that the course gives them a basic understanding of the concept of massive MIMO, as an early insight into the main cutting-edge technology component of next generation wireless networks.

Some feedback brought forward by the students included:

- It has been expressed that we could have been more thorough in the research of equipment that was chosen for the course, since there were some compatibility issues (drivers for the A/D and D/A cards).

- There is a wish that the system should be expanded with more units to allow for a larger scale test system. 16 units is borderline "massive" and with 16 units it is difficult to get good results with 4 units as terminals and the remaining 12 constituting the massive MIMO array. This certainly makes for good future projects in the coming years.

- Many students would like to see how the different parts of a communication system go together in real-world systems more in detail already in earlier courses, to be better prepared for this kind of task. As in most universities, the majority of the regular coursework focuses on "theory", more than practical implementation aspects. 
According to the students, the most important experience from the project was to create an actual, complete communication system, with real hardware. This enabled the students to use much of their knowledge from courses, while also encountering the problems that are always present with physical systems and real hardware. Solving these kinds of problems is an excellent way for the students to enhance their general skills in engineering. At the same time, they are introduced to the next generation communication technology.

Another aspect that the students benefit from during the project course is the opportunity to work in groups with mixed nationalities. Since the course is on the master level, typically exchange students from different countries take the course, in addition to Swedish engineering students. Thus the students get to work in a somewhat international environment, which is a good preparation for future employment as engineers.

Taken together, the students appreciated the opportunity to work practically with a physical system that implements the basic principles of a cutting-edge technology, massive MIMO. They also enjoy working in a very structured manner, following a project model which sets up a well-defined, logical framework for their work. This project is still relatively young, and leaves continued opportunities for improvements in the education and preparation of students for future engineering work.

\section{Appendix: Detailed Description of the Transceiver Device Design}

The setup consisted of a general purpose computer, D/A- and A/D-converter cards, modified active loudspeakers and signal distribution hardware. An overview of this setup is given in Figure 4. 16 channels are used from each of the A/D- and D/A-cards (DA12-16 [13,14] and AD12-64 [15,16] from Contec), one A/D- and D/A-channel per speaker. The speakers are used both as speakers and as microphones. We used small active off-the-shelf speakers (Maxxtro MX-US-08 [17]), where the original power amplifiers are used as such.

The speakers come in pairs, where one serves as master and contains all the electronics, while the other serves as slave and contains only a speaker element.

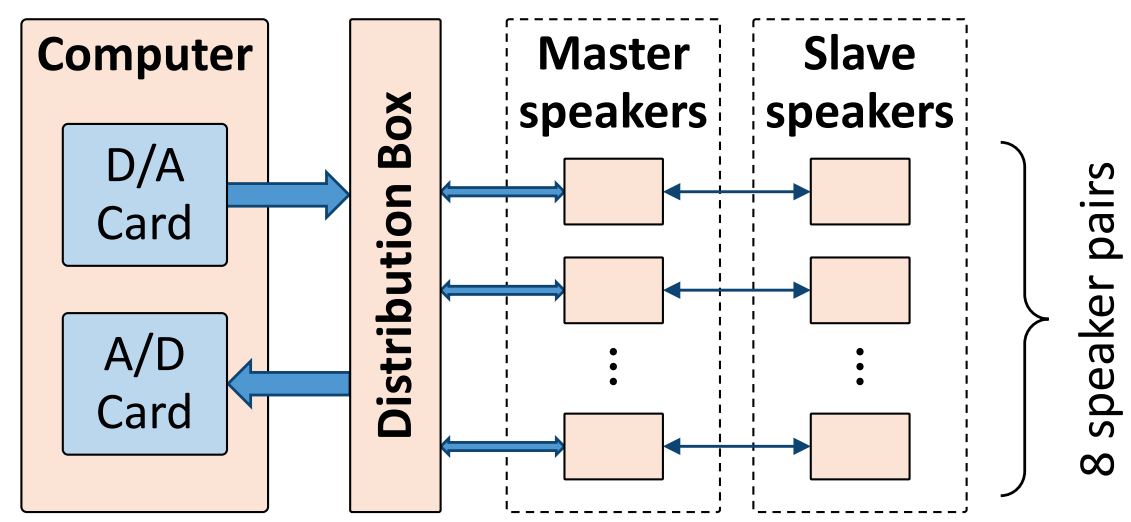

Figure 4. Overview of the setup.

The master speaker was modified to facilitate the selection between two modes: either both the master and the slave operate as loudspeakers, or they operate as microphones. This modification is illustrated in Figure 5. On the detection board, relays are used to switch 
between two operation modes: (i) using the speaker elements of a loudspeaker pair as transmitters, by connecting them to the outputs of the power amplifiers, or (ii) using the speaker elements as microphones, by connecting them to the inputs of the receive chain. This way, the hardware supports half-duplex communication.

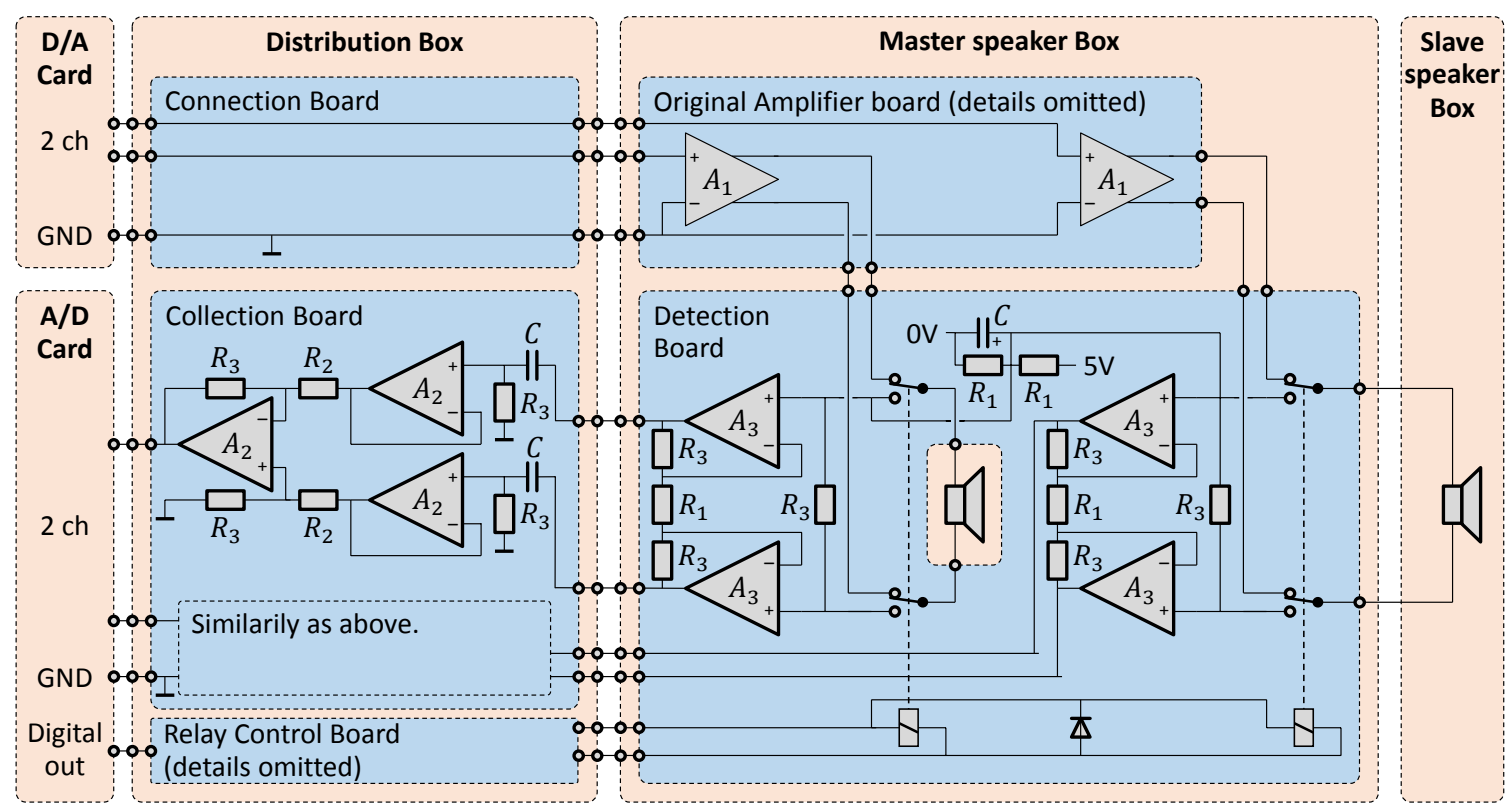

Figure 5. Schematic of the hardware, illustrating the circuitry for one speaker pair. $A_{1}$ is the original power amplifier for one speaker. $A_{2}$ is $1 / 4$ of a TLO74 [19] quad OPamp. $A_{3}$ is $1 / 4$ of a MCP6024 [18] quad low-noise OPamp. Discrete component values: $R_{1}=1 \mathrm{k} \Omega, R_{2}=10 \mathrm{k} \Omega, R_{3}=100 \mathrm{k} \Omega, C=1 \mu \mathrm{F}$.

The receive chain for a single speaker is essentially a traditional instrumentation amplifier, that usually is built around three OPamps and seven resistors to determine the voltage gain, as illustrated in Figure 6. In our implementation (Figure 5) we use five OPamps. The first two OPamps were placed on the circuit board in the master speaker and form a differential-in and differential-out amplifier with a linear voltage gain of 200 (46 dB).

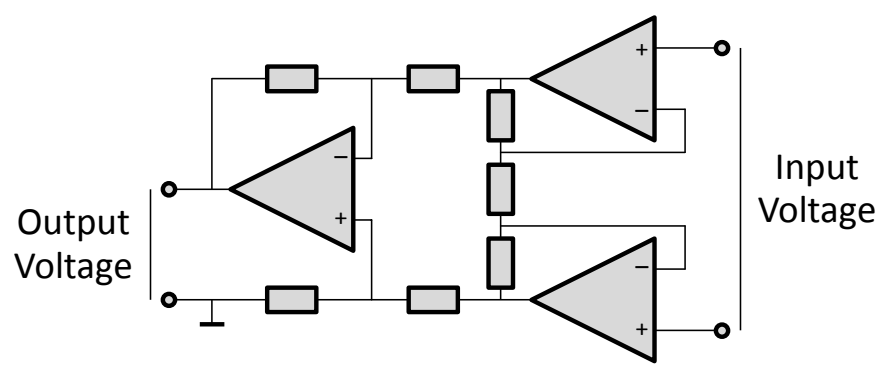

Figure 6. A traditional instrumentation amplifier based on 3 OPamps and 7 resistors.

The signal is then sent via a cable to a distribution box where signals from all speaker pairs are further amplified before they are sent to the A/D-converter card. The signals travel 
differentially from the speakers to the distribution box to counteract capacitively coupled noise. The last three OPamps in the detection signal chain were placed on the collection board in this distribution box together with the corresponding circuits for the other 7 speaker pairs. The first two of those OPamps are used as voltage followers and their main task is to remove inductively coupled noise by breaking the closed circuit that otherwise would have formed. They are also accompanied by RC-links that form first-order HP filters to remove any offset voltage introduced by the OPamps in the speaker. These are the two extra OPamps compared to a standard instrumentation amplifier. The fifth and final OPamp in this signal chain is a differential-in single-ended-out amplifier which provides an additional linear voltage gain of $10(20 \mathrm{~dB})$.

This setup poses special demands on the OPamps of the detection board in the master speaker box. We amplify weak signals, which means that we need low-noise amplifiers on the detection board. Also, the fairly large initial voltage gain (200) demands that the Input Offset Voltage of these first OP-amps is low. Finally, these OP-amps must be able to operate at supply voltage $5 \mathrm{~V}$, since that is what is available in the master speaker box. We use MCP6024 I/P [18], which is a quad low-noise OP-amp that accepts supply voltage from $2.5 \mathrm{~V}$ to $5.5 \mathrm{~V}$ and delivers full output swing. Its Input Offset Voltage is in the range $\pm 500 \mu \mathrm{V}$. With voltage gain 200, this limits the Output Offset Voltage to be in the range $\pm 100 \mathrm{mV}$. The Gain-Bandwidth Product of this OP-amp is $10 \mathrm{MHz}$, so with voltage gain 200, we end up with a bandwidth of $50 \mathrm{kHz}$.

The sampling frequency is determined by the A/D- and D/A-cards. They operate at a maximum of $100 \mathrm{ksamples} / \mathrm{s}$, which is distributed between the used channels. Thus, with 16 channels, the sampling frequency used is 6250 samples/s. The bandwidth $50 \mathrm{kHz}$ of the analog part of the detection signal chain is therefore well sufficient.

While our experience from the particular choice of hardware has been positive, future generations of the experimental setup may use other components, in particular, eliminating the master-slave configuration inherited from the choice of the Maxxtro loudspeaker pairs, for which there is no natural need in our application.

\section{References}

1. T. L. Marzetta, E. G. Larsson, H. Yang and H. Q. Ngo, Fundamentals of Massive MIMO, Cambridge University Press, 2016.

2. Problems to Fundamentals of Massive MIMO [1], including solution manual.

3. The conceive-design-implement-operate (CDIO) official website (http://cdio.org/)

4. J. Vieira, F. Rusek and F. Tufvesson, "Reciprocity calibration methods for massive MIMO based on antenna coupling", 2014 IEEE Global Communications Conference, Austin, TX, 2014, pp. 3708-3712.

5. 5G Researchers Set New World Record For Spectrum Efficiency, IEEE Spectrum, May 2016 (http://bit.ly/1YoxMM7) 
6. J. Vieira, S. Malkowsky, K. Nieman, Z. Miers, N. Kundargi, L. Liu, I. Wong, V. Öwall, O. Edfors and F. Tufvesson, "A flexible 100-antenna testbed for Massive MIMO", IEEE Globecom Workshops, Austin, TX, 2014, pp. 287-293, 2014.

7. Massive MIMO 5G USRP testbed - KU Leuven, the TELEMIC group (http://www.esat.kuleuven.be/telemic/research/NetworkedSystems/infrastructure) massive-mimo-5g)

8. P. Harris, S. Zang, A. Nix, M. Beach, S. Armour and A. Doufexi, "A distributed massive MIMO testbed to assess real-world performance and feasibility", 2015 IEEE 81st Vehicular Technology Conference (VTC Spring), Glasgow, 2015, pp. 1-2.

9. X. Yang, W.-J. Lu, N. Wang, K. Nieman, S. Jin, H. Zhu, X. Mu, I. Wong, Y. Huang and X. You, "Design and implementation of a TDD-Based 128-antenna massive MIMO prototyping system", arXiv:1608.07362.

10. N. Choubey and A. Y. Panah, "Introducing Facebook's new terrestrial connectivity systems - Terragraph and Project ARIES" (https://code.facebook.com/posts/1072680049445290/introducing-facebook-s-newterrestrial-connectivity-systems-terragraph-and-project-aries)

11. Acoustic Massive MIMO Testbed (https://www.youtube.com/watch?v=i1OLEEkiobA)

12. T. Svensson and C. Krysander, Project Model LIPS, Studentlitteratur, 2011.

13. Digital to Analog Output Board for PCl - 16ch type - DA12-16(PCI), Datasheet, Ver. 2.13, Contec, (http://www2.contec.co.jp/prod_data/da1216pci/da1216pci_e.pdf)

14. Digital to Analog Output Board for PCI - DA12-16(PCI) - DA12-8(PCI) - DA12-4(PCI) User's Guide, December 2013 Edition, Contec, (http://www2.contec.co.jp/dl data/LZJ367/LZJ367 131203.pdf)

15. Multi-channel Analog Input Board for PCI - AD12-64(PCI), Datasheet, Ver. 3.13, Contec, (http://www2.contec.co.jp/prod_data/ad1264pci/ad1264pci_e.pdf)

16. 64/16 Channel Analog to Digital Input Board for PCI - AD12-64(PCI) - AD12-16(PCI) User's Guide, December 2013 Edition, Contec, (http://www2.contec.co.jp/dl_data/LZJ371/LZJ371_131202.pdf)

17. Maxxtro Mini Speaker MX-US-08, Datasheet, Maxxtro, (http://www.elfa.spb.ru/uploads/tdpdf/kt856563.pdf)

18. MCP6021/1R/2/3/4, Datasheet, Microchip, 2009, (http://ww1.microchip.com/downloads/en/DeviceDoc/21685d.pdf) 
19. TL07xx Low-Noise JFET-Input Operational Amplifiers, Datasheet, Texas Instruments, June 2015 (http://www.ti.com/lit/ds/symlink/tl074.pdf) 